quantification in polarized macrophages showed that miR-326 is mainly expressed by the M2-type macrophages, and MEFV by the M1-type macrophages. Loss-of-function studies showed that neutralization of miR-326 in M2 macrophages induced the expression of $M E F V$ and CXCL10 while reducing MRC1 expression level. Furthermore, enforced expression of miR-326 in M1 macrophages significantly repressed MEFV expression and induced the production of IL-10.

Conclusions A miR-326/MEFV axis seems to be implicated in macrophage polarization and might explain the observed monocyte versatility in FMF.

Disclosure of Interest None declared.

\section{P073 HIGH LDL LEVELS LESSEN BONE DESTRUCTION DURING ANTIGEN-INDUCED ARTHRITIS BY INHIBITING OSTEOCLAST FORMATION AND FUNCTION} ${ }^{3} \mathrm{I}$ Bot, ${ }^{1} \mathrm{FA}$ van de Loo, ${ }^{1} \mathrm{Ml}$ Koenders, ${ }^{1} \mathrm{PM}$ van der Kraan, ${ }^{1} \mathrm{AB}$ Blom, ${ }^{1} \mathrm{PL}$ van Lent. ${ }^{1}$ Experimental Rheumatology, Radboud university medical center, Nijmegen; ${ }^{2}$ Future Diagnostics Solutions, Wijchen; ${ }^{3}$ Leiden Academic Centre for Drug Research, Leiden University, Leiden, Netherlands

\subsection{6/annrheumdis-2018-EWRR2019.62}

Career situation of first and presenting author Post-doctoral fellow.

Introduction Rheumatoid arthritis (RA) is a chronic inflammatory disease, characterized by severe joint inflammation and bone destruction as the result of increased numbers and activity of osteoclasts. In RA, joint destruction is associated with high levels of low-density lipoprotein (LDL), which in inflammatory environments is oxidized into oxLDL. However, the effects of high (ox)LDL levels on the differentiation and activation of osteoclasts remains elusive.

Objectives Here, we investigated the effects of high LDL and oxLDL levels on osteoclast differentiation.

Methods Antigen-induced arthritis (AIA) was induced in Apoe $e^{-/}$ mice that spontaneously develop high LDL levels. Bone erosion was assessed with histology and numbers of osteoclasts were determined with staining for tartrate-resistant acid phosphatase (TRAP). Numbers of $\mathrm{CD}_{11} \mathrm{~b}^{+} / \mathrm{Ly} 6 \mathrm{C}^{\text {high }}$ and $\mathrm{CD} 11 \mathrm{~b} /$ Ly6C high osteoclast precursors were determined by flow cytometry. In vitro osteoclast differentiation from bone marrow cells was induced with macrophage colony-stimulating factor (M-CSF) and receptor activator of nuclear factor kappa-B (RANK) for 7 days. Gene expression was determined with qPCR.

Results Whereas basal levels of bone resorption were comparable between WT and Apoe $e^{-/}$mice, induction of AIA resulted in significantly lower bone resorption in $A p o e^{-/-}$mice as compared to WT mice, which was associated with lower number of $\mathrm{TRAP}^{+}$osteoclasts along the bone surface. However, the absence of Apoe did not result in altered numbers of osteoclast precursors in the bone marrow of naïve mice, whereas even increased numbers were observed in $A p o e^{-/-}$mice during AIA. Moreover, in vitro osteoclastogenesis showed comparable numbers and mRNA expression of osteoclast markers, such as c-Fms, RANK, NFATc1, DC-STAMP, TRAP, CTR, ClC-7, CAII, Cat $\mathrm{K}$ and MMP-9. Addition of oxLDL, but not LDL, to pre-osteoclasts from day 3 and mature osteoclasts from day 6 of osteoclastogenesis strongly reduced the number of $\mathrm{TRAP}^{+}$ osteoclasts and their resorptive capacity. This was accompanied by a decreased expression of various osteoclast markers.
Interestingly, oxLDL decreased the expression of osteoclast associated receptor (Oscar) and the DNAX adaptor protein-12 encoding gene Tyrobp, which regulate the immunoreceptor tyrosine-based activation motif (ITAM) mediated co-stimulation signaling pathway that is strongly involved in osteoclastogenesis.

Conclusions $A p o e^{-/-}$mice have decreased bone resorption during experimental RA, probably via oxLDL-mediated interference in the co-stimulatory pathway during osteoclastogenesis.

Disclosure of Interest None declared.

\section{P074 THE ALARMIN S100A9 HAMPERS OSTEOCLAST DIFFERENTIATION FROM CIRCULATING PRECURSORS BY REDUCING THE EXPRESSION OF RANK}

${ }^{1} \mathrm{MH}$ van den Bosch, ${ }^{1} \mathrm{I}$ Di Ceglie*, ${ }^{1} \mathrm{AB}$ Blom, ${ }^{2} \mathrm{C}$ Logie, ${ }^{2} \mathrm{JH}$ Martens, ${ }^{3} \mathrm{~J}$ Roth, ${ }^{3} \mathrm{~T}$ Vogl, ${ }^{4} \mathrm{CS}$ Goodyear, ${ }^{1} \mathrm{PM}$ van der Kraan, ${ }^{1} \mathrm{PL}$ van Lent. 'Experimental Rheumatology, Radboud university medical center; ${ }^{2}$ Department of Molecular Biology, Radboud University, Nijmegen, Netherlands; ${ }^{3}$ Institute of Immunology, University of Münster, Münster, Germany; ${ }^{4}$ Institute of Infection, Immunity and Inflammation, University of Glasgow, Glasgow, UK

\subsection{6/annrheumdis-2018-EWRR2019.63}

Career situation of first and presenting author Post-doctoral fellow.

Introduction The alarmin S100A8/A9 is produced in high levels in inflamed synovium during arthritic diseases and has been implicated in sterile inflammation-induced bone resorption. We have previously shown that this alarmin increases the bone-resorptive capacity of mature osteoclasts. However, the effects on osteoclast differentiation remains elusive.

Objectives Here, we investigated the effects of S100A9 on osteoclast differentiation from $\mathrm{CD}_{1}{ }^{+}$circulating precursors.

Methods CD14 ${ }^{+}$monocytes were isolated from buffy coats of healthy donors and differentiated towards osteoclasts with macrophage colony-stimulating factor (M-CSF) and receptor activator of nuclear factor kappa-B (RANK) ligand in the presence or absence of S100A9. Differentiation state of osteoclasts was determined by tartrate-resistant acid phosphatase (TRAP) staining and resorption capacity using hydroxyapatite-likecoated plates. RNA expression was analyzed with RNA sequencing and qPCR. RANK expression was assessed using FACS. Underlying epigenetic programming was studied using chromatin immunoprecipitation. Secretion of pro-/anti-inflammatory mediators was analyzed with Luminex analysis.

Results S100A9 stimulation during monocyte-to-osteoclast differentiation resulted in a strong decrease in the numbers of multinucleated osteoclasts, underlined by a decreased resorptive capacity. The thus differentiated cells showed a high production of pro-inflammatory factors, such as interleukin (IL)$1 \beta$, IL-6, IL-8, and tumor necrosis factor- $\alpha$ (TNF $\alpha$ ) after 16 hour of stimulation. In contrast, at day 4 , the cells showed a decreased expression of the osteoclast-promoting factor $\mathrm{TNF} \alpha$. Interestingly, S100A9 stimulation during the first 16 hour of culture was sufficient to reduce osteoclastogenesis. We observed that within this time frame, S100A9 inhibited the M-CSF-mediated induction of RANK, which associated with changes in various histone marks at the epigenetic level. This S100A9-induced reduction in RANK could be partially reversed by blocking $\mathrm{TNF} \alpha$, but not interleukin-1 (IL-1).

Conclusions Whereas S100A8/A9 was previously shown to stimulate the resorptive capacity of mature osteoclasts, we here show that early S100A9 stimulation impedes monocyteto-osteoclast differentiation via reduction of RANK expression 